\title{
Determinaçáo da CL90 e TL90 do isolado IBCB66 de Beauveria bassiana (Ascomycetes: Clavicipitaceae) para o controle de Rhipicephalus (Boophilus) microplus (Acari: Ixodidae)
}

\author{
Determination of LC 90 and LT 90 of IBCB66 Beauveria bassiana (Ascomycetes: Clavicipitaceae) \\ isolate for Rhipicephalus (Boophilus) microplus (Acari: Ixodidae) control
}

Leila A. G. Barci ${ }^{1 *}$; José Eduardo M. de Almeida²; Adriana H. de Campos Nogueira ${ }^{3}$; Angelo P. do Prado ${ }^{4}$

${ }^{1}$ Instituto Biológico, Centro de Pesquisa e Desenvolvimento de Sanidade Animal - CPDSA

${ }^{2}$ Instituto Biológico, Centro Experimental do Instituto Biológico - CEIB

${ }^{3}$ Agência Paulista de Tecnologia dos Agronegócios - APTA

${ }^{4}$ Departamento de Parasitologia, Universidade Estadual de Campinas - UNICAMP

Recebido em 2 de Outubro de 2008

Aceito em 9 de Setembro de 2009

\section{Resumo}

O presente trabalho teve por objetivo avaliar a patogenicidade e a virulência do isolado IBCB66 de Beauveria bassiana para larvas de Rhipicephalus (Boophilus) microplus. O isolado IBCB66 foi utilizado como padrão, com a finalidade de determinar a CL50 (Concentração Letal), CL90, TL50 (Tempo Letal) e TL90. O isolado IBCB66 foi testado em seis concentraçôes diferentes $\left(5 \times 10^{6}, 10^{7}, 5 \times 10^{7}, 10^{8}, 5 \times 10^{8} \mathrm{e} 10^{9}\right)$ para determinar a porcentagem de mortalidade. A

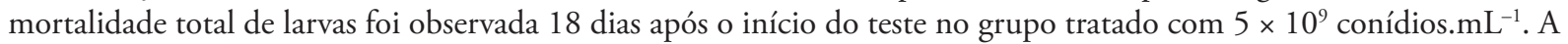

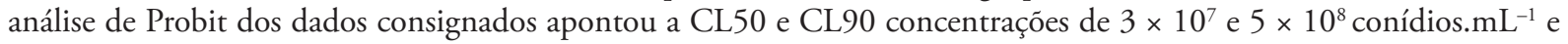
para TL50 e TL90, foram 10 e 16 dias, respectivamente.

Palavras-chave: Bovinos, Controle Biológico, Fungo entomopatogênico, Carrapato.

\begin{abstract}
The objective of this research was to evaluate the pathogenicity and the virulence of the IBCB66 isolate of Beauveria bassiana on infected larvae of Rhipicephalus (Boophilus) microplus. The IBCB66 fungus strain was used as standard isolates of $B$. bassiana against $R$. (B.) microplus larvae. The larval bioassay tests using the IBCB66 isolate were carried out to determine the (Lethal Concentration) LC50, LC90, (Lethal Time) LT50 and LT90. The IBCB66 fungus strain was tested at six different concentrations $\left(5 \times 10^{6}, 10^{7}, 5 \times 10^{7}, 10^{8}, 5 \times 10^{8}\right.$ and $10^{9}$ conidia.mL $\left.\mathrm{mL}^{-1}\right)$ to determine the percentage of larval mortality. In addition, a Probit analysis was also performed. Total larval mortality was observed eighteen days after the beginning of the test in the group treated with $5 \times 10^{9}$ conidia. $\mathrm{mL}^{-1}$. The LC50 and LC90 were $3 \times 10^{7}$ and $5 \times 10^{8}$ respectively and the LT50 and LT90 were 10 and 16 days.
\end{abstract}

Keywords: Cattle, Biological Control, Entomopathogenic fungus, Tick.

\section{Introdução}

O carrapato Rhipicephalus (Boophilus) microplus foi introduzido nos países tropicais e subtropicais por meio das importaçóes de bovinos na época da colonizaçáo (WHARTON, 1974). As condiçôes climáticas de nosso país, amplamente favoráveis ao desenvolvimento do parasita, permitiram que populaçóes de carrapatos se estabelecessem em praticamente todo o território nacional.

\footnotetext{
*Autor para correspondência: Leila Aparecida Gardiman Barci

Centro de Pesquisa e Desenvolvimento de Sanidade Animal - CPDSA,

Instituto Biológico, Av. Conselheiro Rodrigues Alves 1.252,

CEP 04.014-002, São Paulo - SP, Brasil

e-mail: barci@biologico.sp.gov.br
}

Os prejuízos causados anualmente à bovinocultura nacional estáo relacionados ao grande potencial de veiculação de micro-organismos patogênicos, mortalidade e desenvolvimento letárgico de animais, principalmente os de raças europeias, queda nos sistemas produtivos de leite, carne e couro e, consequentemente, baixa agregação de valor aos produtos e altos gastos com insumos veterinários (PENNA, 1990).

Atualmente, essa situação vem se agravando com o desenvolvimento da resistência desses artrópodos aos diferentes grupos químicos nas diversas regiôes do país (MENDES, 2005). Como consequência, o número de pesquisas realizadas em busca de métodos alternativos para o controle de carrapato tem crescido 
sobremaneira. Os trabalhos publicados estão focados na busca de opçôes que possam ser implementadas individualmente ou contribuir na composição de sistemas de controle integrado, a fim de minimizar os problemas ocasionados por esses ectoparasitas (BARCI, 1997).

Presentemente, a maioria das pesquisas produzidas, com o objetivo de empregar agentes microbianos no controle de carrapatos, concentra-se na utilização de fungos entomopatogênicos (SAMISH et al., 2004)

Os fungos apresentam vários fatores altamente vantajosos que os tornam uma excelente opçáo para o controle de ectoparasitas. São patógenos de largo espectro, capazes de atacar insetos e ácaros, promovendo epizootias naturais; infectam diferentes estágios de desenvolvimento dos hospedeiros, como ovos, larvas, pupas, ninfas e adultos, característica desejável e particularmente observada nos diferentes grupos de artropodos. Algumas estirpes são muito virulentas e a maioria é altamente especializada. Os principais tipos de formas de dispersáo - os conídios e esporos - possuem alta capacidade de disseminaçáo horizontal, podendo ser propagados por locais muito distantes. Além disso, estáo amplamente distribuídos no ambiente e são facilmente isolados de amostras de solos, insetos e ácaros infectados (ALVES, 1998). Por causa da ampla utilização na área agrícola, encontram-se presentes nas principais coleçóes de fungos entomopatogênicos do Brasil (Instituto Biológico, ESALQ, EMBRAPA). A coleção do Instituto Biológico conta com grande número de isolados, os quais são amplamente utilizados no combate a pragas agrícolas (ALVES, 1998).

Nesse grupo de micro-organismos, as espécies Metarbizium anisopliae e Beauveria bassiana são apontadas pela literatura como as mais promissoras para utilização como agentes microbianos (SAMISH, et al., 2004).

Tendo em vista a necessidade de estudos referentes à utilização do controle biológico em infestações por carrapatos em bovinos, o presente trabalho teve como objetivo verificar, in vitro, a virulência do isolado IBCB66 do fungo Beauveria bassiana, inoculado sob a forma de suspensão conidial sobre larvas não alimentadas de Rhipicephalus (Boophilus) microplus. E ainda, calcular as concentraçôes letais 50 e 90 (CL50, CL90) e observar o tempo de ação dessa estirpe, utilizando os parâmetros auferidos como padróes em experimentos de seleçáo de isolados do fungo para o controle de carrapatos de bovinos.

\section{Material e Métodos}

Os testes experimentais foram conduzidos no Instituto Biológico/APTA nos laboratórios de Parasitologia Animal (Centro de Pesquisa e Desenvolvimento de Sanidade Animal), em São Paulo, Controle Biológico de Pragas (Centro Experimental do Instituto Biológico), em Campinas, e no Polo Regional de Desenvolvimento Tecnológico dos Agronegócios do Leste Paulista (Monte Alegre do Sul) - APTA Regional.

Os carrapatos utilizados no presente trabalho procederam de animais mestiços, de produção leiteira, nascidos e criados em Monte Alegre do Sul e infestados experimentalmente com amostra da região. O município localiza-se no Estado de São Paulo, a $22^{\circ} 43^{\prime} \mathrm{S}$ e $46^{\circ} 37^{\prime} \mathrm{O}, 740 \mathrm{~m}$ de altitude, apresentando solo da classe podzólico vermelho amarelo.

Fêmeas ingurgitadas foram coletadas diretamente do animal, levadas ao laboratório, onde foram separadas e identificadas quanto ao gênero e espécie, segundo chave de Aragão e Fonseca (1961), lavadas com água destilada, secas em papel macio e mantidas sob $26,5^{\circ} \mathrm{C}$ de temperatura e $80 \%$ de umidade relativa.

Os ovos e as larvas deles eclodidas foram acondicionados em frascos de penicilina sob as mesmas condiçóes controladas. Nos bioensaios, foram utilizadas larvas infestantes com idade entre 15 e 18 dias.

A escolha do isolado de B. bassiana IBCB66, como padrão, foi realizada com base na virulência do entomopatógeno, constatada em bioensaios desenvolvidos em laboratório e na eficácia do isolado, quando utilizado no controle microbiano de ácaros e/ou insetos em condiçôes de campo (BATISTA-FILHO, 2004).

Essa amostra encontra-se depositada no Banco de Microrganismos Entomopatogênicos "Oldemar Cardim Abreu" do Laboratório de Controle Biológico/Centro Experimental do Instituto Biológico, localizado em Campinas - SP, mantida em freezer a $-12{ }^{\circ} \mathrm{C}$, na forma de conídios puros.

O cultivo do isolado do fungo foi obtido por meio do plaqueamento em meio de ágar batata dextrose $(\mathrm{ABD})$. Antes de receber o inóculo, o meio foi autoclavado a $120{ }^{\circ} \mathrm{C}$ durante 30 minutos. Após proceder a inoculação, o material foi mantido em sala com temperatura e umidade controladas $\left(26 / 29^{\circ} \mathrm{C}\right.$ e $80 \%$ UR e fotofase de 12 horas), onde permaneceu por aproximadamente 15 dias, quando apresentou coloraçáo branca ou ligeiramente amarelada (esporulação). Após atingir a esporulaçáo, o material foi retirado da sala de crescimento e acondicionado a $-13^{\circ} \mathrm{C}$.

As suspensões de conídios foram preparadas a partir dos fungos retirados do meio $\mathrm{ABD}$ (suspensão mãe), o qual foi raspado com auxílio de uma alça de metal e diluído em água destilada e espalhante adesivo Tween $80^{\circ}$.

A partir da suspensão-mãe, foram preparadas as seguintes concentraçôes de conídios do fungo. $\mathrm{mL}^{-1}: 5 \times 10^{6}, 10^{7}, 5 \times 10^{7}$, $10^{8}, 5 \times 10^{8} \mathrm{e} 10^{9}$. A quantificaçáo dos conídios foi feita por meio de contagem direta ao microscópio com auxílio de câmara de Neubauer (ALVES; MORAES, 1998).

Para o desenvolvimento do experimento foi utilizada a metodologia descrita por Barci e Nogueira (2006), que permite a observação diária de indivíduos de um mesmo tratamento, pelo menos por 20 dias, sem que haja alteraçáo significativa nos percentuais de mortalidade do grupo controle.

Antes de iniciar o bioensaio, frascos de penicilina contendo aproximadamente 10.000 larvas foram abertos e as larvas ativas, potencialmente infestantes, subiram e ficaram agrupadas na borda do frasco. Com auxílio de um pincel de cerdas macias, as larvas foram coletadas, colocadas em placa de Petri contendo disco de papel de filtro, imersas em $3 \mathrm{~mL}$ da suspensão a ser testada e cobertas novamente com disco de papel de filtro formando um bloco único no modelo de sanduíche.

Após 10 minutos em contato com a suspensão, o sanduíche foi retirado da placa de Petri e colocado sobre uma "almofada" feita de folhas de papel de filtro recortadas em tamanho de papel A4. Os discos de papel de filtro contendo as larvas banhadas pela suspensão de fungo foram separados e as larvas em movimento 
foram aspiradas para dentro de uma pipeta, tipo Pasteur, fechada com tecido de organza (malha de $50 \mu \mathrm{m}$ ) na extremidade mais larga. A aspiração foi realizada com auxilia de Bomba de Vácuo utilizando-se pressão de $1 \mathrm{Lb} \mathrm{Pol}^{-2}$. Após a aspiração as pipetas foram seladas na extremidade oposta à tela, separadas por tratamento e mantidas durante todo o bioensaio em câmara climatizada a $27^{\circ} \mathrm{C}$ e $80 \%$ de UR em recipiente perfurado.

O grupo testemunha recebeu como tratamento água destilada com espalhante adesivo Tween $80^{\circ}$.

O delineamento experimental utilizado foi inteiramente casualizado, constando de 5 repetiçóes por tratamento, sendo utilizadas 20 larvas em cada repetição, perfazendo total de 100 indivíduos por tratamento.

Para determinação dos parâmetros relativos à concentração letal 50 e 90 (CL50 e CL90) e do tempo letal 50 e 90 (TL50 e TL90), adotados como valores de referência, foram realizadas avaliaçóes diárias do $1^{\circ}$ dia pós-infecção até o dia em que foi constatada mortalidade total de um dos tratamentos.

Foram determinados valores relativos à mortalidade acumulada e corrigida, calculada de acordo com a Equação 1 sugerida por Abbott (1925).

$$
\% \text { mortalidade corrigida }=\frac{\begin{array}{c}
\text { números de vivos controle }- \\
\text { número vivos tratado }
\end{array}}{\text { números de vivos controle }} \times 100
$$

Os resultados obtidos foram analisados levando-se em conta as diferentes concentraçóes de conídios. $\mathrm{mL}^{-1}$ do isolado IBCB66 de $B$. bassiana testadas (variável independente), e a porcentagem de mortalidade de larvas de $R$. (B.) microplus obtidas nos diferentes tratamentos (variável dependente). Os dados foram transformados em raiz quadrada de $(\mathrm{x}+0,5)$, submetidos à análise de variância (ANOVA) e, a posteriori, ao teste de Tukey para comparação de médias, a 5\% de significância global.

Para determinar a concentração letal 90 (CL90) e o tempo letal 90 (TL90), os dados obtidos foram submetidos à análise de Probites POLO PC Software (LEORA SOFTWARE, 1987).

\section{Resultados}

A mortalidade total das larvas de todas as parcelas do mesmo tratamento ocorreu no grupo tratado com suspensão de $10^{9}$ conídios. $\mathrm{mL}^{-1}$ no décimo oitavo dia após a infecção (DAI).

Os resultados obtidos nos seis tratamentos com as diferentes concentraçôes $\left(5 \times 10^{6}\right.$ a $10^{9}$ conídios. $\left.\mathrm{mL}^{-1}\right)$ revelaram percentuais de mortalidade entre 19,6 e $100 \%$.

A análise estatística dos dados indicou que houve diferença significante entre os percentuais de mortalidade obtidos nos grupos tratados com as concentraçôes $5 \times 10^{6}, 1 \times 10^{7}$, tanto entre si como em relação às demais suspensôes do bioensaio. Os percentuais de mortalidade obtidos nos tratamentos, a partir da concentração $5 \times 10^{7}$, apresentaram valores não significativos, quando comparados dois a dois, entre uma concentraçáo e a subsequente. Dessa forma, os percentuais de mortalidade de larvas tratadas com suspensóes contendo $5 \times 10^{7}$ e $1 \times 10^{8}$ apresentaram diferenças significativas em relação aos demais, porém não diferiram entre si; o mesmo fato pode ser constatado entre os tratamentos
$1 \times 10^{8}$ e $5 \times 10^{8}$ e observado entre as suspensóes $5 \times 10^{8} \mathrm{e}$ $1 \times 10^{9}$ conídios. $\mathrm{mL}^{-1}$ (Tabela 1$)$.

Ao se comparar o valor dos limites unilaterais de F, apontados pela tabela adaptada a partir de resultados de Pearson (GOMES, 1988), ao nível de significância de 5\% de probabilidade (aproximadamente 2,2) com o obtido $(101,53)$, foi constatado que os resultados consignados são altamente significativos.

Todos os tratamentos realizados diferiram do grupo-controle que apresentou porcentagem de mortalidade muito baixa (3\%).

Os valores referentes à mortalidade total foram muito próximos àqueles calculados para a mortalidade corrigida pela fórmula de Abbott (1925).

Por meio da análise de Probites foram estabelecidos como valores referentes à CL50 e CL90, respectivamente, as concentraçóes $3 \times 10^{7}$ e $5 \times 10^{8}$. Utilizando-se o mesmo método para obtenção do TL50 e TL90, foram determinados os seguintes dias: 10 e 16 DAI (Tabelas 2 e 3 ).

Os percentuais de mortalidade obtidos no grupo tratado com suspensão de $5 \times 10^{8}$ conídios. $\mathrm{mL}^{-1}$ do isolado IBCB66 de B. bassiana, entre o período de 10 a $18 \mathrm{DAI}$, foram comparados entre si, conforme registros expressos na Tabela 4 .

Os percentuais de mortalidade acumulada no período variaram entre 39 e $82 \%$, e a mortalidade corrigida apresentou índices entre 38,4 e $81,4 \%$.

A análise de variância indicou que não houve diferença entre os percentuais de mortalidade ocorridos nos dias 10 e 11 DAI.

Os índices de mortalidade consignados no $12^{\circ}$ DAI divergiram daqueles apontados nos dois dias anteriores, porém náo apresentaram diferença significativa dos obtidos nos três dias consecutivamente posteriores.

Os registros de mortalidade para o período entre o $12^{\circ} \mathrm{e}$ $18^{\circ}$ DAI sugeriram que não há discordância estatística entre os percentuais de mortalidade obtidos nos blocos que englobam os dias 12, 13 e 14. A mesma observação vale para os dias 13, 14 e 15; 14,15 e 16; 15, 16 e 17 e, finalmente, 16,17 e 18 .

O coeficiente de variaçáo do bioensaio - 3,41 - foi considerado muito baixo e o valor de $\mathrm{F}(57,75)$ altamente significativo, quando comparado ao estabelecido por Pearson (GOMES, 1988), para nível de significância de 5\% (2,2).

Tabela 1. Mortalidade acumulada de larvas de Rhipicephalus (Boophilus) microplus tratadas com diferentes concentraçôes de Beauveria bassiana isolado IBCB66, 18 dias após infecção, em condições de laboratório

\begin{tabular}{|c|c|c|c|}
\hline \multirow{2}{*}{$\begin{array}{l}\text { Concentraçáo } \\
\left(\text { conídios.mL } L^{-1}\right)\end{array}$} & \multicolumn{2}{|c|}{ Mortalidade (\%) } & \multirow{2}{*}{$\begin{array}{c}\text { Média e erro } \\
\text { padráo }\end{array}$} \\
\hline & Acumulada & Corrigida & \\
\hline Testemunha & 3 & - & $0,6 \pm 0,8944^{a}$ \\
\hline $5 \times 10^{6}$ & 22 & 19,6 & $4,4 \pm 1,5166^{b}$ \\
\hline $1 \times 10^{7}$ & 40 & 38,1 & $8,0 \pm 2,4495^{c}$ \\
\hline $5 \times 10^{7}$ & 59 & 57,7 & $11,8 \pm 0,8366 \mathrm{~d}$ \\
\hline $1 \times 10^{8}$ & 71 & 70,1 & $14,2 \pm 1,4832 \mathrm{de}$ \\
\hline $5 \times 10^{8}$ & 82 & 81,4 & $16,4 \pm 1,1402$ ef \\
\hline $1 \times 10^{9}$ & 100 & 100 & $20,0 \pm 0,0000$ \\
\hline
\end{tabular}
(Temperatura $26,5^{\circ} \mathrm{C}$ e Umidade Relativa $80 \%$ ).

Dados originais seguidos do erro padrão da média; Médias seguidas da mesma letra na coluna não diferem estatisticamente entre si pelo teste de Tukey (erro global $\alpha=0.05$ ); Teste ANOVA de um fator; $\mathrm{CV}=8,66$; $\mathrm{F}=101,56$; Dados transformados em Raiz quadrada de $(\mathrm{x}+0,5)$ para fins de análise estatística. 
Tabela 2. Valores referentes às concentraçóes letais 50 e 90 do isolado IBCB66 de Beauveria bassiana, para o controle in vitro de larvas de Rhipicephalus (Boophilus) microplus, determinados pela análise de Próbites.

\begin{tabular}{lcc}
\multicolumn{1}{c}{ Parâmetro } & CL90 & CL50 \\
\hline Limites fiduciais (95\%) & $3,016892 \mathrm{E}+08<$ CL90 $<7,001103 \mathrm{E}+08$ & $2,098922 \mathrm{E}+07<$ CL50 $<3,721379 \mathrm{E}+07$ \\
Valor de a & $-1,243$ & $-1,243$ \\
Valor de r2 & 0,8630 & 0,8630 \\
Valor de G & 0,0223 & 0,0223 \\
Valor de b & 0,837 & 0,837 \\
Intervalos de confiança de b & $0,764393<\mathrm{b}<0,909666$ & $0,764393<\mathrm{b}<0,909666$ \\
Qui-quadrado & 22,3911 & 22,3911 \\
Grau de liberdade & 28 & 28 \\
Concentraçáo Eficaz & $4,379724 \mathrm{E}+08\left(4,4 \times 10^{8}\right)$ & $2,834667 \mathrm{E}+07\left(2,8 \times 10^{7}\right)$ \\
\hline
\end{tabular}

Tabela 3. Valores referentes aos tempos letais 50 e 90 do isolado IBCB66 de Beauveria bassiana para o controle de larvas in vitro de Rhipicephalus (Boophilus) microplus, determinados pela análise de Próbites.

\begin{tabular}{lcc}
\multicolumn{1}{c}{ Parâmetro } & TL90 & TL50 \\
\hline Limites fiduciais (95\%) & $15,17789<$ TL90 $<16,11585$ & $10,26573<$ TL50<10,9868295 \\
Valor de a & 2,933 & 2,933 \\
Valor de r ${ }^{2}$ & 0,9171 & 0,9171 \\
Valor de G & 0,0050 & 0,0050 \\
Valor de b & 0,216 & 0,216 \\
Intervalos de confiança de b & $0,2072504<\mathrm{b}<0,2241534$ & $0,2072504<\mathrm{b}<0,2241534$ \\
Qui-quadrado & 62,2136 & 62,2136 \\
Grau de liberdade & 88 & 88 \\
Tempo Eficaz (Dias) & $15,621(16$ dias $)$ & $10,566958(10$ dias $)$ \\
\hline
\end{tabular}

Tabela 4. Mortalidade acumulada e corrigida de larvas de Rhipicephalus (Boophilus) microplus tratadas in vitro com suspensão de $5 \times 10^{8}$ conídios do isolado IBCB66 de Beauveria bassiana no período de 10 a 18 DAI (dias após infecção).

\begin{tabular}{cccc}
\hline \multirow{2}{*}{ DAI } & \multicolumn{2}{c}{ Mortalidade } & Média e erro padráo \\
\cline { 2 - 3 } & Acumulada & Corrigida & \\
\hline 10 & 39 & 38,4 & $7,8 \pm 1,9235^{\mathrm{a}}$ \\
11 & 46 & 45,5 & $9,2 \pm 2,0494^{\mathrm{a}}$ \\
12 & 60 & 59,6 & $12,0 \pm 2,6457^{\mathrm{b}}$ \\
13 & 65 & 64,6 & $13,0 \pm 2,5495^{\mathrm{bc}}$ \\
14 & 67 & 66,7 & $13,4 \pm 2,1909^{\mathrm{bcd}}$ \\
15 & 71 & 70,4 & $14,2 \pm 2,5884 \mathrm{cde}$ \\
16 & 75 & 74,2 & $15,0 \pm 1,4142$ def \\
17 & 80 & 79,4 & $16,0 \pm 1,4142$ ef \\
18 & 82 & 81,4 & $16,4 \pm 1,1402 \quad \mathrm{f}$ \\
\hline
\end{tabular}

Dados originais seguidos do erro padrão da média; Médias seguidas da mesma letra na coluna năo diferem estatisticamente entre si pelo teste de Tukey (erro global $\alpha=0.05$ ); $C V=3,41$; Teste ANOVA de um fator; D.M.S. $5 \%=0,54754$-; D.M.S. $1 \%=0,66469$.

Os dados de mortalidade acumulada e corrigida, foram muito próximos, quando comparados dois a dois para o mesmo dia de tratamento.

\section{Discussão}

Os resultados obtidos permitiram verificar que, nas concentraçóes entre $5 \times 10^{6}$ a $10^{9}$ conídios. $\mathrm{mL}^{-1}$, o isolado IBCB66 de $B$. bassiana apresenta potencial patogênico para controlar larvas de $R$. (B.) microplus em condiçóes de laboratório. Entretanto, apenas concentraçóes iguais ou superiores a $5 \times 10^{8}$ conídios. $\mathrm{mL}^{-1}$, que promoveram $83 \%$ de mortalidade de larvas, mostraram eficácia necessária para possível utilização a campo.

Larvas de $R$. (B.) microplus tratadas com suspensóes do entomopatógeno apresentaram percentuais de mortalidade inversamente proporcional à concentração de conídios. $\mathrm{mL}^{-1}$, dados concordantes com aqueles apontados por Paião et al. (2001) e Fernandes et al. (2006).

Consultando registros da literatura nacional, foi possível verificar que muitos isolados de B. bassiana utilizados em experimentaçóes conduzidas com larvas e outros ínstares de carrapatos de importância médico-veterinária apresentam índices que não diferem muito dos verificados neste trabalho.

Em relação à CL50, os dados obtidos neste trabalho $2,8 \times 10^{7}$ - são próximos àqueles mencionados por Bittencourt et al. (1999) - 1,8 $\times 10^{6}$ - que avaliou a patogenicidade in vitro do isolado Bb747 sobre larvas do carrapato $A$. nitens em 
experimentaçôes conduzidas com suspensôes que variaram entre $10^{5}$ a $10^{8}$ conídios. $\mathrm{mL}^{-1}$.

Monteiro et al. (2001), ao testar os isolados CG17, EP01 e 986 de $B$. bassiana sobre larvas de Anocentor nitens, determinaram as respectivas CL50 $\left(1,8 \times 10^{7}, 1,6 \times 10^{6}\right.$ e $2,5 \times 10^{7}$ conídios. $\left.\mathrm{mL}^{-1}\right)$, quando trabalharam com quatro concentraçóes que variaram entre $10^{5}$ e $10^{8}$ conídios. $\mathrm{mL}^{-1}$.

Analisando os dados referentes à CL90 do isolado IBCB66, pode-se observar que esses valores estão muito próximos daqueles apontados por Bittencourt et al. (1997) que, em ensaios com fêmeas tratadas com suspensóes de dois isolados de Beauveria bassiana, determinaram como CL90 a concentração de $1,15 \times 10^{9}$ conídios. $\mathrm{mL}^{-1}$ para o isolado 747 e $5,95 \times 10^{8}$ conídios. $\mathrm{mL}^{-1}$ para o 986 .

Examinando o tempo letal obtido na presente pesquisa, baseando-se na análise estatística dos dados estabelecidos pelo teste de Tukey a 5\% de significância, pode-se inferir que o TL50 do isolado IBCB66 para controlar larvas de $R$. (B.) microplus in vitro com suspensóes de $5 \times 10^{8}$ conídios. $\mathrm{mL}^{-1}$, foi de 11 ou 12 dias e o TL90 de 16, 17 ou 18 dias.

Ainda com relação aos tempos letais 50 e 90, não há como estabelecer comparaçóes entre os dados aqui consignados com os apontados na literatura, pois a maior parte dos registros é resultante de delineamentos experimentais onde o dia de avaliação do bioensaio foi previamente estabelecido e não houve observação diária da mortalidade acumulada.

Fernandes et al. (2003) e Fernandes et al. (2006), em ensaios conduzidos com larvas de $R$. (B.) microplus tratadas com diferentes isolados de $B$. bassiana, efetuaram as avaliaçóes dos bioensaios no décimo dia após o tratamento. Paião et al. (2001) detectaram os índices de mortalidade de larvas de $R$. (B.) microplus tratadas com três isolados de $B$. bassiana no $20^{\circ} \mathrm{DAI}$

Monteiro et al. (1998), ao estudar a ação de isolados de B. bassiana sobre larvas de Rhipicephalus sanguineus, realizaram as observaçóes 10 dias após o tratamento. O mesmo procedimento foi empregado por Souza et al. (1999) e Bittencourt et al. (1999), ao avaliarem, respectivamente, o efeito de $B$. bassiana para larvas de Amblyomma cajennense e Anocentor nitens.

Reis et al. (2001) demonstraram a ação in vitro de isolados de $B$. bassiana em ninfas e adultos de Amblyomma cajennense por intermédio de observaçóes realizadas 15 dias após o tratamento de larvas ou depois da ecdise de adultos.

Em relação ao tempo necessário para que o isolado IBCB66 promova controle efetivo de larvas de $R$. (B.) microplus (16 a 18 dias em condiçôes ótimas), é pertinente considerar a biologia do carrapato. Dependendo das condiçóes climáticas, as larvas se tornam infestantes por um período de até vinte dias após a eclosão, e o tempo de vida livre dessa fase gira em torno de 28 a 51 dias, podendo ficar até seis meses sem se alimentar. $\mathrm{O}$ ciclo evolutivo bastante longo permite a exposição dessa fase por tempo suficiente para que haja, em condiçóes de campo, grande chance de colonização desse estágio de desenvolvimento (ROCHA, 2009).

De qualquer forma, os tempos necessários para o estabelecimento de controles efetivos são bem diferentes daqueles observados em tratamentos com produtos químicos.

É preciso ter em mente que, no controle de ectoparasitas, a estratégia de utilização de entomopatógenos difere daquela estabelecida para inseticidas químicos. $\mathrm{O}$ controle químico promove a redução rápida e temporária dos prejuízos econômicos causados por infestações por artrópodos. Os produtos microbianos reduzem as populaçóes das pragas mais lentamente, sendo os resultados mais duradouros que os proporcionados pelos agrotóxicos.

\section{Conclusóes}

O isolado IBCB66 do fungo Beauveria bassiana foi eficaz para o controle in vitro de larvas de $R$. (B.) microplus.

A concentraçáo letal 50 (CL50) do isolado IBCB66 de $B$. bassiana para o controle in vitro do carrapato $R$. (B.) microplus foi de $3 \times 10^{7}$ conídios. $\mathrm{mL}^{-1}$.

O tempo letal 50 (TL50) do isolado IBCB66 de B. bassiana, com o propósito de controlar in vitro larvas do carrapato $R$. (B.) microplus, foi de 10 dias, podendo ser as avaliaçóes realizadas no $10^{\circ}$ ou $11^{\circ}$ DAI.

A CL90 do isolado IBCB66 de B. bassiana para o controle in vitro de larvas do carrapato $R$. (B.) microplus foi de $5 \times 10^{8}$ conídios. $\mathrm{mL}^{-1}$.

O tempo letal 90 (TL90) do isolado IBCB66 de B. bassiana com o propósito de se controlar in vitro larvas do carrapato $R$. (B.) microplus foi de 16 dias, podendo ser as avaliaçóes realizadas 16, 17 ou 18 DAI.

\section{Referências}

ABBOTT, W. S. A method for computing the effectiviness of insecticides. Journal of Economic Entomology, v. 18, n. 15, p. 265-267, 1925.

ALVES, S. B. Fungos entomopatogênicos. In: Controle Microbiano de Insetos. 2 ed. Piracicaba: FEALQ, 1998. p. 289-381.

ALVES, S. B.; MORAES, S. A. Quantificação de inóculo de patógenos de insetos. In: ALVES, S. B. (Ed.). Controle Microbiano de Insetos. 2 ed. Piracicaba: FEALQ, 1998. p. 765-777.

ARAGÃO, H.; FONSECA, F. Notas de Ixodologia. Memórias do Instituto Oswaldo Cruz, v. 59, n. 2, p. 115-129, 1961. (VIII, Lista e chave para os representantes da fauna ixodológica brasileira).

BARCI, L. A. G. Controle biológico do carrapato dos bovinos Boophilus microplus (Acari, Ixodidae) no Brasil. Arquivos do Instituto Biológico, v. 64, n. 1, p. 95-101, 1997.

BARCI, L. A. G.; NOGUEIRA, A. H. C. Método para avaliação de mortalidade de larvas de Boophilus microplus (Canestrini, 1887) submetidas a tratamentos com produtos carrapaticidas. Arquivos do Instituto Biologico, v. 73, n. 1, p. 105-109, 2006.

BATISTA-FILHO, A. Comunicação pessoal. 2004.

BITTENCOURT, V. R. E. P. et al. Avaliação da eficácia in vitro de dois isoldados do fungo entomopatogênico Beauveria bassiana (Bals.) Vuill. em fêmeas ingurgitadas de Boophilus microplus (Canestrini, 1887) (Acari: Ixodidae). Revista Brasileira de Parasitologia Veterinária, v. 6, n. 1, p. 49-52, 1997.

BITTENCOURT, V. R. E. P. et al. Ação dos fungos Beauveria bassiana (Balsamo)Vuillemin, 1912 e Metarbizium anisopliae (Metschnikoff,1879) Sorokin, 1883 sobre larvas do carrapato Anocentor nitens (Acari: Ixodidae). Parasitologia Al Dia, v. 23, n. 3/4, p. 82-86, 1999. Disponível em: <http:// www.scielo.cl/scielo.php?pid=S0716-07201999000300003\&script=sci arttext>. Acesso em: Agosto 2008. 
FERNANDES, E. K. et al. Beauveria bassiana isolated from engorged females and tested against eggs and larvae of Boophilus microplus (Acari: Ixodidae). Journal of Basic Microbiology, v. 43, n. 5, p. 393-398, 2003.

FERNANDES, E. K. et al. Study on morphology, pathogenicity, and genetic variability of Beauveria bassiana isolates obtained from Boophilus microplus tick. Parasitology Research, v. 98, n. 4, p. 324-332, 2006.

GOMES, F. P. A estatística moderna na pesquisa agropecuária. Piracicaba: POTAFÓS, 1988. 160 p.

LEORA SOFTWARE. A user's guide to probit or logit analysis. Berkeley, CA: LeOra Software, 1987.

MENDES, M. C. Resistência do carrapato Boophilus microplus (Acari: Ixodidae) aos piretróides e organofosforados e o tratamento carrapaticida em pequenas fazendas. Campinas, 2005. 122p. Tese (Doutorado em Parasitologia) - Universidade Estadual de Campinas - UNICAMP.

MONTEIRO, S. G. et al. Pathogenicity under laboratory conditions of the fungi Beauveria bassiana and Metarhizium anisopliae on larvae of the tick Rhipicephalus sanguineus (Acari: Ixodidae). Revista Brasileira de Parasitologia Veterinária, v. 7, n. 2, p. 113-116, 1998.

MONTEIRO, S. G.; BITTENCOURT, V. R. E. P.; DAEMON, E. Avaliação in vitro da patogenicidade dos isolados CG17, EP01 e 986 do fungo Beauveria bassiana (balsamo) Vuillemin sobre larvas do carrapato Anocentor nitens (Neumann, 1897) (Acari: Ixodidae). Revista Brasileira de Ciência Veterinária, v. 8, n. 3., p. 144-146, 2001.
PAIÁO, J. C. V.; MONTEIRO, A. C.; KRONKA, S. N. Susceptibility of the cattle tick Boophilus microplus (Acari: Ixodidae) to isolates of the fungus Beauveria bassiana. World Journal of Microbiology \& Biotechnology, v. 17, n. 3, p. 245-251, 2001.

PENNA, V. M. Boophilus microplus: a resistência genética do hospedeiro como forma de controle. Cadernos Técnicos da Escola de Veterinária da Universidade Federal de Minas Gerais, v. 4, p. 3-65, 1990.

REIS, R. C. S. et al. Ação in vitro dos fungos Beauveria bassiana (Bals) Vuill e Metarhizium anisopliae (Metsch) Sorok sobre ninfas e adultos de Amblyomma cajennense (Fabricius, 1787) (Acari: Ixodidae). Arquivo Brasileiro de Medicina Veterinária e Zootecnia, v. 53, n. 5, p. 544-547, 2001.

ROCHA, C. M. B. M. Importância do carrapato Boophilus microplus, Canestrini, 1887 (Acarina, Ixodidae) no processo produtivo do leite. Lavras: Universidade Federal de Lavras. Disponível em: <http://www. editora.ufla.br/BolTecnico/pdf/bol_35.pdf>. Acesso em: Abril 2009.

SAMISH, M.; GINSBERG, H.; GLAZER, I. Biological control of ticks. Parasitology, v. 129, p. 389-403, 2004. (Suplemento).

SOUZA, E. J.; REIS, R. C. S.; BITTENCOURT, V. R. E. P. Evaluation of in vitro effect of the fungi Beauveria bassiana and Metarhizium anisopliae on eggs and larvae of Amblyomma cajennense. Revista Brasileira de Parasitologia Veterinária, v. 8, n. 2, p. 127-131, 1999.

WHARTON, R. H. The current status and prospects for the control of Inoxid ticks with especial emphasis on Boophilus microplus. Bulletin de L'Office International des Epizooties, v. 81, n. 1/2, p. 65-85, 1974. 